\title{
Improving low-cost
}

\section{inertial-measurement-unit (IMU)-based motion tracking accuracy for a biomorphic hyper-redundant snake robot}

\author{
Weixin Yang ${ }^{*}$ (D), Alexandr Bajenov and Yantao Shen
}

\begin{abstract}
This paper develops and experimentally validates a 3D-printed snake robot prototype. Its structure is designed to allocate limited room for each functional module (including an external power module, battery power module, the wireless control and transmission module and some detective sensors), so as to ensure the snake robot works in different environments. In order to control and track the snake robot, a low-cost MEMS-IMU (micro-electro-mechanical systems inertial measurement unit)-based snake robot motion tracking system is developed. Three algorithms (low-pass filter, baseline calibration, and Kalman filter) are used to eliminate noise from IMU's acceleration data, thus minimizing the noise influence to tracking accuracy. Through signal processing, the IMU acceleration data can be effectively used for motion tracking. The result from the video tracking software is employed as a reference for comparison, so as to evaluate the motion tracking algorithm efficiency. The comparison results demonstrate high efficiency of the proposed IMU-based motion tracking algorithm.
\end{abstract}

Keywords: Snake robot, 3D printing, IMU, Motion tracking

\section{Background}

Biological snake has a cord-like body with many links (bones) which can form diverse locomotion modes with many functions [1]. Snake is one of the most successfully evolved species, which has lived on the earth for 300 million years because of its high adaptation to the environment. Different locomotion modes also have various functions. Some snakes have the ability to change their movement mode immediately when entering into a new environment. Because of the snake's versatility, the study of snake locomotion mode promises many future applications such as space exploration, environmental monitoring, transportation, and surveillance.

According to the research on the biological snake, the snake-inspired robot has extremely high redundancy with many degrees of freedom, with its body on

*Correspondence: weixiny@nevada.unr.edu

Electrical and Biomedical Engineering, University of Nevada, Reno, N. Virginia St, Reno, NV 89557, USA the ground and kinetically stable. This feature makes the snake easily adapt to irregular terrain. Based on this high adaptability, the snake robot can be adopted where human beings cannot go to carry out tasks. The snake's locomotion modes can be categorized into following four types: (1) serpentine locomotion; (2) rectilinear locomotion; (3) rolling locomotion; and (4) sidewinding locomotion. In this paper, two locomotion modes are developed. The serpentine movement is the most typical locomotion mode, which has been widely observed in almost all species of snakes. According to Hirose's [2] research on the biological snake, the research starts with generating the serpentine curve for the snake robot [3]. To test additional locomotion performance of the robot, sidewinding is further investigated and performed on the developed robot.

The snake robot is designed to collect information in some challenging environments, such as forest, cave, building ruins, or even underwater. Most of these environments are GPS-denied, leading to the difficulties in 
tracking and controlling snake robot. Hence, it is necessary to consider and understand the efficiency of each locomotion mode in different environments based on collected terrain information. Many studies on object's attitude determination with MEMS-IMU have been performed for many years. MEMS-IMU-based tracking technology [4] is widely applied in many fields, such as detection of unconstrained walking [5], pedestrian tracking in indoor environments [6, 7].

However, IMU-based tracking system calculates the position by double integration of measured acceleration, and with double integration, calculation errors grow rapidly, thus the system being not reliable for a long-time tracking without the aid of GPS or another reference system. Hence, this research focuses on accuracy of IMUbased tracking system by using an improving acceleration estimation method.

To eliminate the effects of accelerometer errors, most of the papers adopt some sensor fusion algorithms, among which the Kalman filter is the most commonly used. In [8-10], standard Kalman filter, unscented Kalman filter (UKF), and extended Kalman filter (EKF) are employed in high data rate signal processing. EKF has lower accuracy; however, UKF requires more computational time.

The paper is organized as follows. In "Custom-built snake robot" section, a customized snake robot is built through 3D printing. "Snake robot trajectory tracking" section presents the motion tracking algorithm along with the three signal processing algorithms including Butterworth low-pass filter, baseline calibration, and Kalman filter. After data processing, the acceleration data can be used for motion tracking. The conclusion and discussion are given in "Conclusion" section.

\section{Custom-built snake robot}

The snake robot joint structure is modularly designed, with the upper part and the lower part being designed separately. Therefore, different chassis can be used based on the environment. The snake robot is composed of eight identical modular units. The joint model size is $50 \mathrm{~mm}$ in diameter and $144 \mathrm{~mm}$ in length. The volume of the snake robot's body is widened by increasing the body width, and thus the integrated electronic system can be installed for controlling. This snake robot has two different bending axes connected alternately at $90{ }^{\circ} \mathrm{C}$. This allows the snake robot to have 3D motion because of the 2 degrees of freedom for each joint (total 16 degrees of freedom). Moreover, each joint can be controlled separately. The hollowed-out grids structure on robot's body increases the structure flexibility, thus enhancing its coefficient of restitution and making the robot run efficiently. The 3D-printed snake robot is shown in Fig. 1.

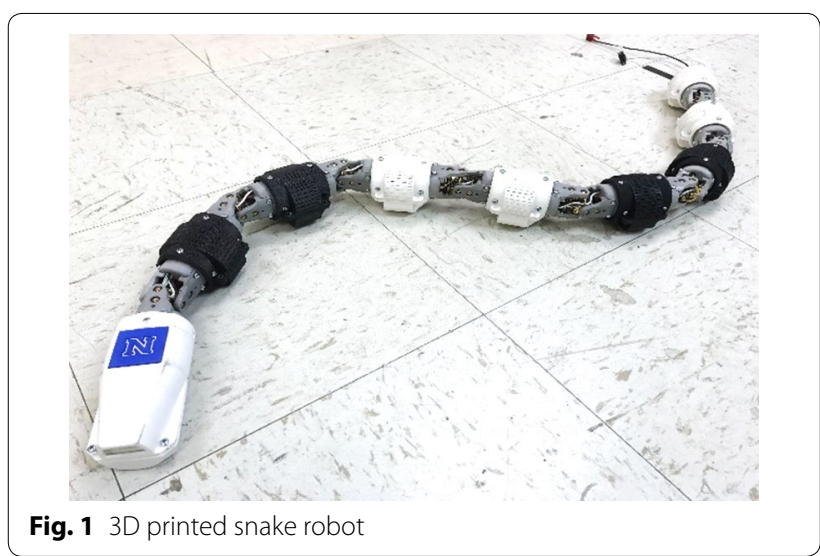

\section{Snake robot trajectory tracking Motion tracking algorithm}

Snake robot displacement can be obtained by the double integral of its acceleration. A low-cost IMU is installed in the head of the snake robot, in order to collect its realtime acceleration data at three axes with high frequency. At the same time, all acceleration data are transferred to a computer for calculation. Then the robot's instant speed curve and accumulated space trajectory are graphed on the screen. Acceleration data are processed by MATLAB. The basic algorithm is shown in Fig. 2.

Assume sampling starts at time $t_{0}$. According to the integration principle, the calculation relationship among displacement $s(t)$, velocity $v(t)$, and acceleration $a(t)$ in the continuous-time domain is:

$$
\begin{aligned}
& s(t)=\int_{t_{0}}^{t} v(t) \mathrm{d} t+s\left(t_{0}\right) \\
& v(t)=\int_{t_{0}}^{t} a(t) \mathrm{d} t+v\left(t_{0}\right)
\end{aligned}
$$

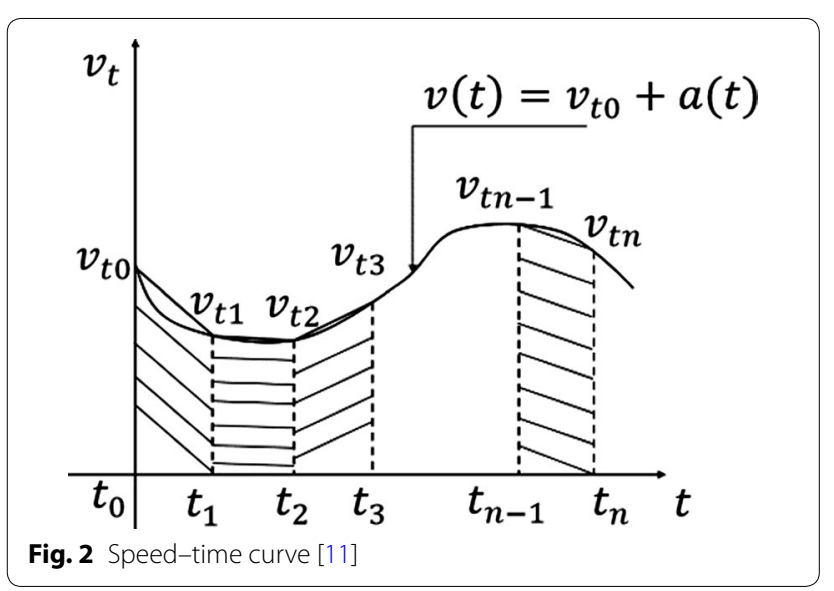


$s\left(t_{0}\right)$ is accumulative displacement from 0 to $t_{0}, v\left(t_{0}\right)$ is the system instant motion velocity at $t_{0}$.

Since the outputs of the IMU are groups of discrete data, the time-speed curve is decomposed as numbers of a right-angled trapezoid. Initials $\left(t_{0}\right)=0$, the displacement $s(t)$ is presented as:

$$
\begin{aligned}
s(t)= & \int_{t_{0}}^{t} v(t) \mathrm{d} t=\frac{v\left(t_{0}\right)+v\left(t_{1}\right)}{2}\left(t_{1}-t_{0}\right) \\
& +\frac{v\left(t_{2}\right)+v\left(t_{1}\right)}{2}\left(t_{2}-t_{1}\right)+\cdots+\frac{v\left(t_{n}\right)+v\left(t_{n-1}\right)}{t_{n}-t_{n-1}}
\end{aligned}
$$

$t_{1}-t_{0}=t_{2}-t_{1}=\cdots=t_{n}-t_{n-1}=\Delta t$, which is the sampling time interval, when $n>1$,

$$
s(t)=\sum_{k=1}^{n} \frac{v\left(t_{k}\right)+v\left(t_{k-1}\right)}{2} \cdot \Delta t
$$

In the discrete domain, the Eq. (4) is modified as:

$$
s[n]=\sum_{k=1}^{n} \frac{v[k]+v[k-1]}{2} \cdot \Delta t
$$

when $n>1$, in the discrete domain

$$
\begin{aligned}
v[n]= & \sum_{k=1}^{n} \frac{a[k]+a[k-1]}{2} \cdot \Delta t \\
= & v[0]+\frac{1}{2}(a[0]+a[n]) \cdot \Delta t+(a[1])+a[2] \\
& +\cdots+a[n-1]) \cdot \Delta t \\
s[n]= & \sum_{k=1}^{n} \frac{v[k]+v[k-1]}{2} \cdot \Delta t \\
= & \frac{1}{2}(v[0]+v[n]) \cdot \Delta t+(v[1])+v[2] \\
& +\cdots+v[n-1]) \cdot \Delta t
\end{aligned}
$$

Then the object's displacement in one axis is presented as:

$$
\begin{aligned}
s[n]= & n \cdot v[0] \cdot[(n-1) \cdot a[1]+(n-2) \cdot a[2] \\
& +\cdots+v[n-1]] \cdot \Delta t^{2}+\frac{1}{4}(a[0]+a[n]) \cdot \Delta t^{2}
\end{aligned}
$$

Through Eq. (8), it is easy to calculate the displacement after obtaining the initial velocity and acceleration from the IMU. However, based on this equation, the calculation is very complicated for large $n$ which leads to huge burden to the system since the system needs to allocate huge memory sources to store the acceleration data from time number 0 to $n$. Furthermore, the system has to repeat all the previous acceleration calculations to update the object's motion displacement. Thus, iteration is applied to simplify the calculation.

From Eq. (6)

$$
\begin{gathered}
v[n]-v[n-1]=\frac{a[n]+a[n-1]}{2} \cdot \Delta t \\
s[n]-s[n-1]=\frac{v[n]+v[n-1]}{2} \cdot \Delta t \\
=v[n-1] \cdot \Delta t+\frac{1}{4}(a[n-1]+a[n]) \cdot \Delta t^{2}
\end{gathered}
$$

Equations (9) and (10) are modified as:

$$
\begin{aligned}
& v[n]=v[n-1]+\frac{a[n]+a[n-1]}{2} \cdot \Delta t \\
s[n] & =s[n-1]+\frac{v[n]+v[n-1]}{2} \cdot \Delta t \\
& =s[n-1]+v[n-1] \cdot \Delta t+\frac{1}{4}(a[n-1]+a[n]) \cdot \Delta t^{2}
\end{aligned}
$$

From Eqs. (11) and (12), the object's instant speed $v[n]$ and motion displacement $s[n]$ can be calculated recursively. The IMU has the acceleration outputs at $X-, Y$ - and $Z$ - axis. Based on Eq. (11), the object's instant speed at $X-, Y$ - and $Z$-axis can be presented as:

$$
\begin{aligned}
& v_{x}[t]=\bar{v}_{x}[t-\Delta t]+\frac{\bar{a}_{x}[t]+a_{x}[t-\Delta t]}{2} \cdot \Delta t \\
& v_{y}[t]=\bar{v}_{y}[t-\Delta t]+\frac{\bar{a}_{y}[t]+a_{y}[t-\Delta t]}{2} \cdot \Delta t \\
& v_{z}[t]=\bar{v}_{z}[t-\Delta t]+\frac{\bar{a}_{z}[t]+a_{z}[t-\Delta t]}{2} \cdot \Delta t
\end{aligned}
$$

The object's instant speed in 3D space at time $t$ can be calculated as:

$$
v[t]=\bar{v}_{x}[t]+\bar{v}_{y}[t]+\bar{v}_{z}[t]
$$

Similarly, the object's motion displacement at $X-, Y$ - and $Z$-axis is presented as:

$$
\begin{aligned}
s_{x}[t]= & s_{x}[t-\Delta t]+v_{x}[t-\Delta t] \cdot \Delta t \\
& +\frac{1}{4}\left(a_{x}[t-\Delta t]+a_{x}[T]\right) \cdot \Delta t^{2} \\
s_{y}[t]= & s_{y}[t-\Delta t]+v_{y}[t-\Delta t] \cdot \Delta t \\
& +\frac{1}{4}\left(a_{y}[t-\Delta t]+a_{y}[T]\right) \cdot \Delta t^{2}
\end{aligned}
$$




$$
\begin{aligned}
s_{z}[t]= & s_{z}[t-\Delta t]+v_{z}[t-\Delta t] \cdot \Delta t \\
& +\frac{1}{4}\left(a_{z}[t-\Delta t]+a_{z}[T]\right) \cdot \Delta t^{2}
\end{aligned}
$$

During time $t-\Delta t$, the object's space motion displacement is:

$s_{\Delta}[t]=\sqrt{\left(s_{x}[t]-s_{x}[t-\Delta t]\right)^{2}+\left(s_{y}[t]-s_{y}[t-\Delta t]\right)^{2}+\left(s_{z}[t]-s_{z}[t-\Delta t]\right)^{2}}$

So at time $t$, the object's space coordinate is $\left(s_{x}[t], s_{y}[t], s_{z}[t]\right)$. In the space coordinate system, the object's trajectory can be plotted by connecting all the space coordinate points from $t_{0}$ to $t_{n}$.

\section{Noise processing of tracking system}

Since the tracking system is based on the double integral of acceleration, errors of IMU measurements cause incorrect integration results and accumulated bias during integration. Even minimal measurement errors will result in huge calculation errors, leading to the low accuracy in tracking results. This paper proposes several methods to filter the acceleration data, so as to make the acceleration data available to integrate. The flow diagram for data processing is shown in Fig. 3.

\section{Low-pass filter}

IMU has noisy signals when stationary. To analyze the impact of noise, 16,000 stationary data are collected, i.e. 4000 points for each axis plus 4000 starting points. Then, calculate the average acceleration value of each axis, finally, subtracting the average value from every single data for each axis, thus getting the acceleration bias of each axis which is shown in Fig. 4.

From Fig. 4, the acceleration vibrates randomly with a maximum value of $0.05 \mathrm{~m} / \mathrm{s}^{2}$. That means the object has maximum $0.05 \mathrm{~m}$ shift when it keeps stationary. Therefore, the errors have a great influence on the tracking system accuracy. To eliminate noise, it is necessary to analyze the signal properties in the frequency domain as shown in Fig. 5.

After Fourier transform of the acceleration data, it is clear in the above picture of Fig. 5 that the noise of each axis is mainly around $50 \mathrm{~Hz}$ in stationary. From the lower picture of Fig. 5, the valid acceleration data are mainly

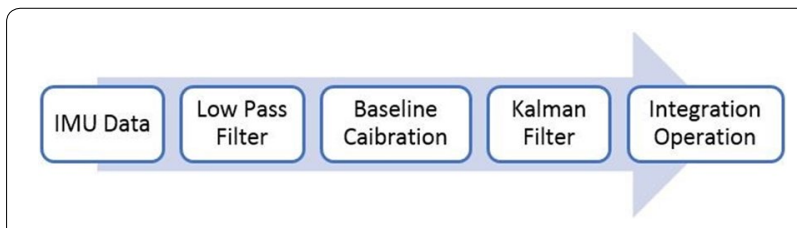

Fig. 3 Data processing flow diagram

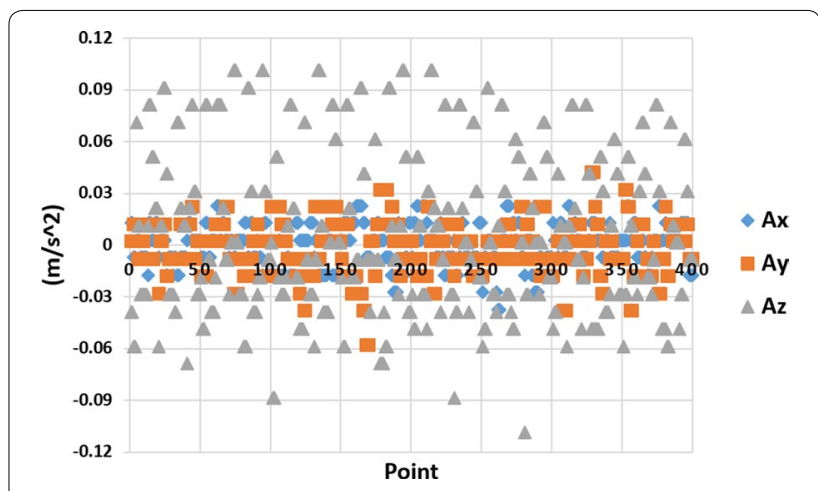

Fig. 4 Acceleration data when stationary

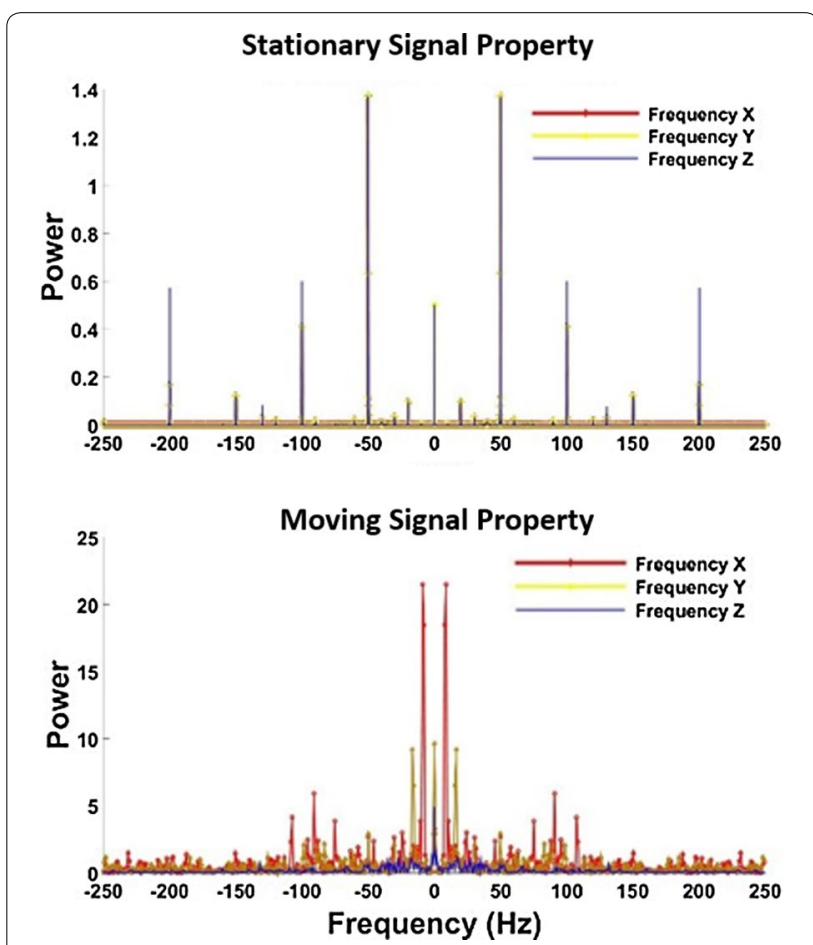

Fig. 5 Signal properties in frequency domain

around $10 \mathrm{~Hz}$ for the $X$-axis and $15 \mathrm{~Hz}$ for the $Y$-axis when moving.

By programming IMU, the data output frequency is 50 $\mathrm{Hz}$. A hardware RC low-pass filter is employed between IMU output terminal and wireless transmission chip, so as to reduce high-frequency component. Then by using Butterworth low-pass filter, most of the high-frequency noises are inhibited. The signal decay of the Butterworth low-pass filter is slow but smooth. In this paper, the sampling rate is $500 \mathrm{~Hz}$; the passband corner frequency is 15 $\mathrm{Hz}$; the stopband corner frequency is $50 \mathrm{~Hz}$; the maximum passband ripple is $3 \mathrm{~dB}$; and the minimum attenuation is $60 \mathrm{~dB}$. 
The time-domain graph and spectrogram of acceleration after Butterworth low-pass filtering of the stationary snake robot are shown in Figs. 6 and 7 respectively.

It is clear in Fig. 7, there is noise only in the passband, and its amplitude is very small.

\section{Baseline calibration}

After low-pass filter, the acceleration data can be used for integration. Before integrating the data, the baseline shift cannot be ignored.

The snake moves forward in a line and then stays stationary. After low-pass filter, only the stationary acceleration data of each axis are collected and plotted as shown in Fig. 8.

The baseline shift in the $X$-axis is about $-0.3 \mathrm{~m} / \mathrm{s}^{2}$, i.e. the $X$-axis movement when tracking is $-0.3 \mathrm{~m} / \mathrm{s}$ even if the snake robot is stationary. Similarly, the baseline shift in the $Y$-axis is about $0.3 \mathrm{~m} / \mathrm{s}^{2}$, i.e. the $Y$-axis movement is $0.3 \mathrm{~m} / \mathrm{s}$ when stationary. When including the baseline shift is in the integration, the cumulative errors will have a significant influence on the system accuracy.

The absolute difference of acceleration presents the relative change of acceleration data. When the snake robot

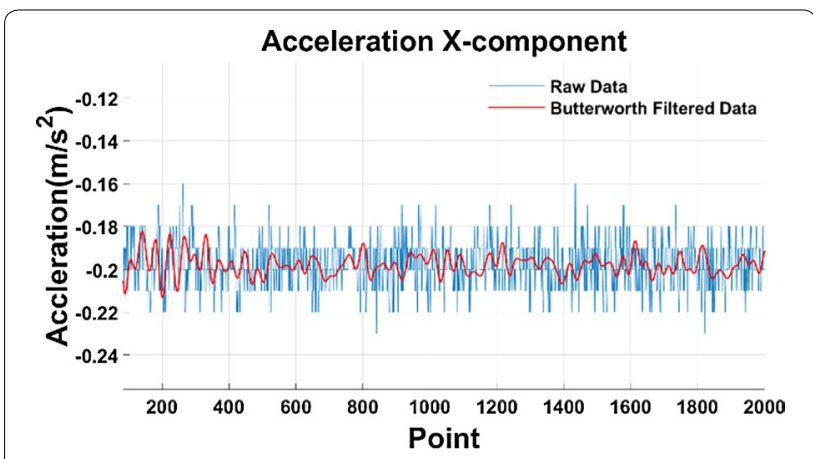

Fig. 6 Filtered acceleration data

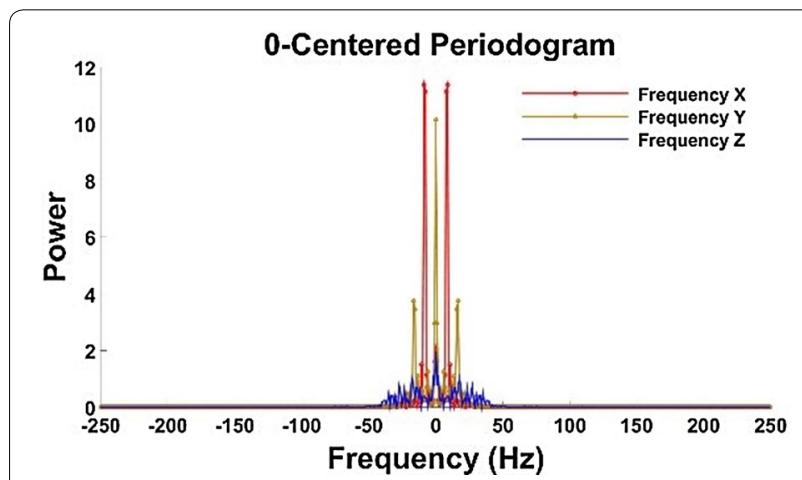

Fig. 7 Spectrogram of moving acceleration after Butterworth lowpass filter

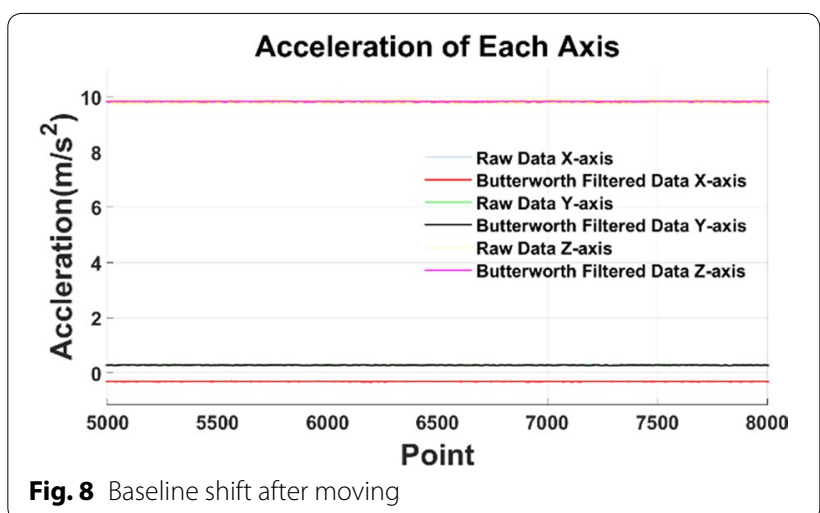

moves fast, the absolute difference becomes relatively big. Also, the absolute difference can present the noise oscillation when the snake robot maintains stationary. MATLAB is used to plot the absolute difference distribution of acceleration, which is shown in Fig. 9.

The above statistic shows that the data oscillation in $X$-axis and $Y$-axis is within 0.05 , while in the $Z$-axis direction, the oscillation is in a wider range of 0.1. Table 1 shows the probability of absolute difference within the range when the snake robot keeps stationary.

The results show the big probability of the absolute difference is within 0.1 when the snake robot is stationary. To compare with the moving acceleration data, the same method is employed. The statistic of oscillation distribution in moving state is shown in Table 2.

The distribution of absolute difference for motion data is concentrated on a smaller interval comparing with stationary data, which has been verified by repeated experiments.

Putting 1000 data from $Y$-axis in one group to calculate the probability of range within $[0,0.05]$. Five groups are repeated, the results of which are shown in Table 3.

From Table 3, the data verify the previous conclusion that the probability of the absolute difference within $[0,0.05]$ in stationary is much higher than that of in moving state. To calibrate the baseline, every 100 data (i.e. data in $0.2 \mathrm{~s}$ ) are taken for the statistic. The statistic shows whether the probability of the absolute difference within $[0,0.05]$ is higher than 0.95 . If so, then the snake robot is stationary. Then the average value of this group data is used as the new average value to shift the acceleration data, i.e., adding or subtracting the compensation value from acceleration data (depending on the sign of acceleration data). At the same time, once the data show that the snake robot is stationary, the value of acceleration data is set to zero.

The baseline calibration results are shown in Fig. 10. The bias after baseline calibration is zero when the snake robot is stationary. 


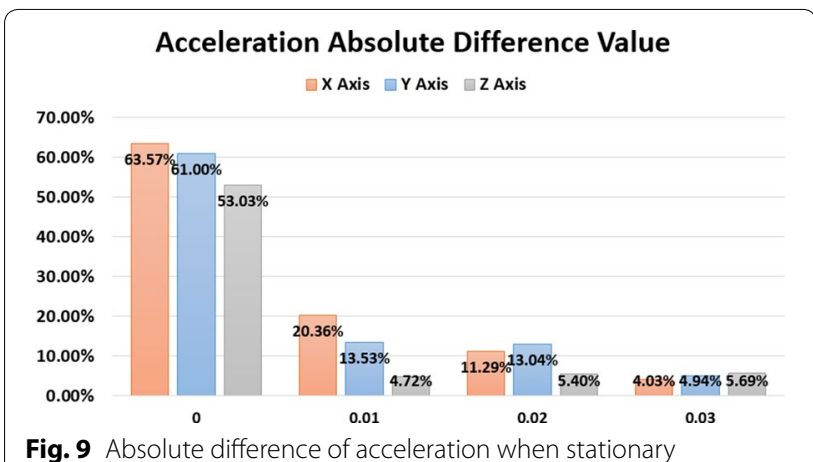

Fig. 9 Absolute difference of acceleration when stationary

Table 1 Statistic of oscillation distribution when stationary

\begin{tabular}{lll}
\hline $\boldsymbol{X}$-axis $<\mathbf{0 . 0 5}$ & $\boldsymbol{Y}$-axis $<\mathbf{0 . 0 5}$ & $\boldsymbol{Z}$-axis $<\mathbf{0 . 1}$ \\
\hline 1 & 0.9418 & 0.9548 \\
\hline
\end{tabular}

Table 2 Statistical analysis of oscillation distribution when moving

\begin{tabular}{lll}
\hline $\boldsymbol{X}$-axis $<\mathbf{0 . 0 5}$ & $\boldsymbol{Y}$-axis $<\mathbf{0 . 0 5}$ & $\boldsymbol{Z}$-axis $<\mathbf{0 . 1}$ \\
\hline 0.0839 & 0.0704 & 0.0124 \\
\hline
\end{tabular}

Table 3 Statistical analysis of oscillation distribution when moving and stationary

\begin{tabular}{llllll}
\hline Probability & Group 1 & Group 2 & Group 3 & Group 4 & Group 5 \\
\hline Stationary & 0.9418 & 0.9854 & 0.9712 & 0.9755 & 0.9587 \\
Moving & 0.0704 & 0.0839 & 0.0655 & 0.0814 & 0.0716 \\
\hline
\end{tabular}

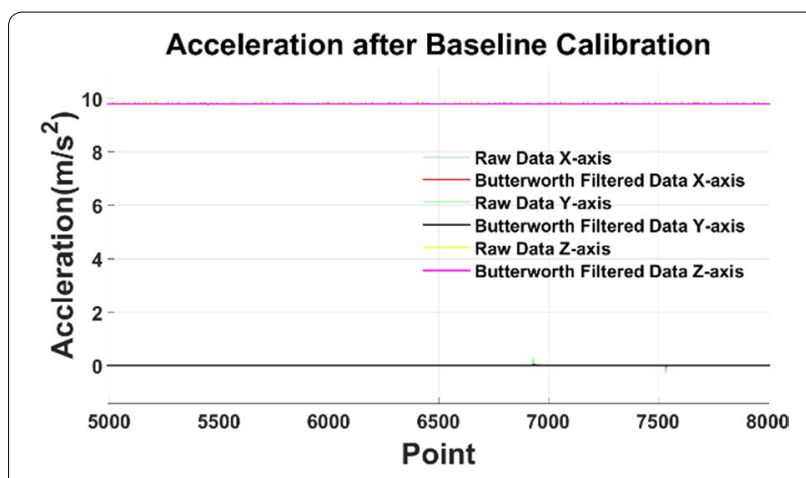

Fig. 10 Baseline calibration result

\section{Kalman filter}

To eliminate random errors, the Kalman filter is applied to the tracking system, thus filtering the acceleration data. The Kalman filter is a set of mathematical equations that provide an efficient computational (recursive) state estimation, so as to minimize the mean squared error [12].

The random process to be estimated can be modeled in the form:

$$
x_{k+1}=\phi_{k} x_{k}+w_{k}+B_{k} u_{k}
$$

when the process state vector is:

$$
x_{k}=\left[\text { Acce_x }, A c c e \_y, A c c e \_z\right]
$$

The initial process state vector is the initial set of acceleration data. The observation (measurement) of the process occurs at discrete points in time by the linear relationship. $\phi_{k}$ is the state transition matrix. $H_{k}$ is the measurement matrix at time $t_{k}$. The initial estimation error covariance is manually selected as $P_{k}^{-}$, where

$$
\begin{gathered}
z_{k}=H_{k} x_{k}+v_{k} \\
\phi_{k}=\left[\begin{array}{lll}
1 & 0 & 0 \\
0 & 1 & 0 \\
0 & 0 & 1
\end{array}\right] H_{k}=\left[\begin{array}{lll}
1 & 0 & 0 \\
0 & 1 & 0 \\
0 & 0 & 1
\end{array}\right] P_{k}^{-}=\left[\begin{array}{ccc}
0.05 & 0 & 0 \\
0 & 0.05 & 0 \\
0 & 0 & 0.05
\end{array}\right]
\end{gathered}
$$

Entering the data into standard Kalman filter, recursive loop consists of Eqs. (24), (25), (26), (27) and (28).

$$
\begin{aligned}
& \hat{x}_{k}=\hat{x}_{k}^{-}+K_{k}\left(z_{k}-H_{k} \hat{x}_{k}^{-}\right) \\
& K_{k}=P_{k}^{-} H_{k}^{T}\left(H_{k} P_{k}^{-} H_{k}^{T}+R_{k}\right)^{-1} \\
& P_{k}=\left(I-K_{k} H_{k}\right) P_{k}^{-} \\
& \hat{x}_{k+1}^{-}=\phi_{k} \hat{x}_{k} \\
& P_{k+1}^{-}=\phi_{k} P_{k} \phi_{k}^{T}+Q_{k}
\end{aligned}
$$

Now all the data and equations are ready for the Kalman filter loop. Once entering the loop, it will run to ad infinitum. The data are transferred by the wireless chip to the computer serial port, and then MATLAB will read the data for Kalman filter algorithm.

\section{Snake robot locomotion analysis}

In the laboratory environment, IMU chip is installed in the head of the snake robot which moves along a straight line. The filtered acceleration data are shown in Fig. 11. It clearly shows in the picture that the highfrequency component of the acceleration is eliminated and the random noise is minimized. The signal curve becomes smooth and available to integrate. The IMU tracking result of snake robot's Serpentine locomotion is shown in Fig. 12. It indicates that the serpentine locomotion has the highest efficiency because the snake 


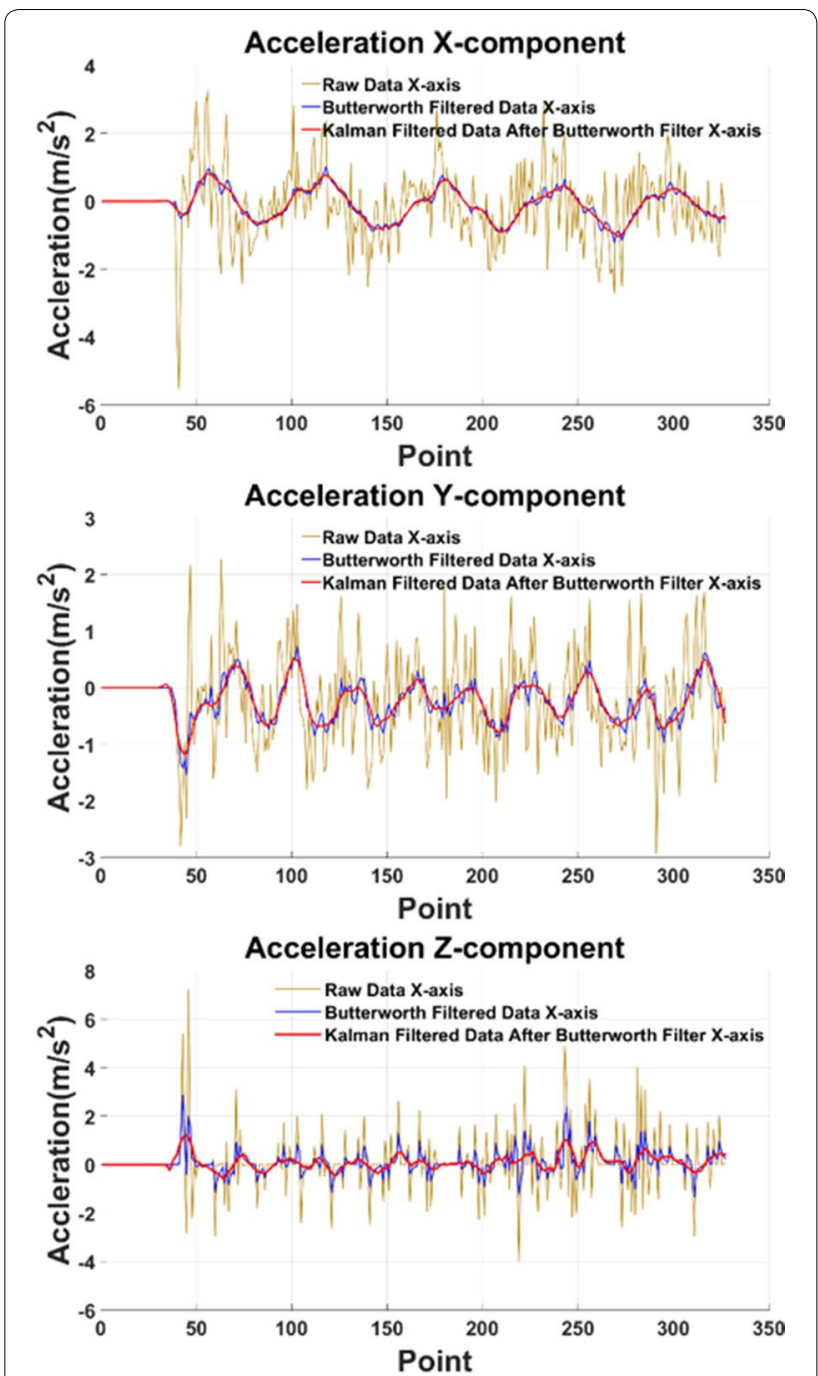

Fig. 11 Snake robot acceleration

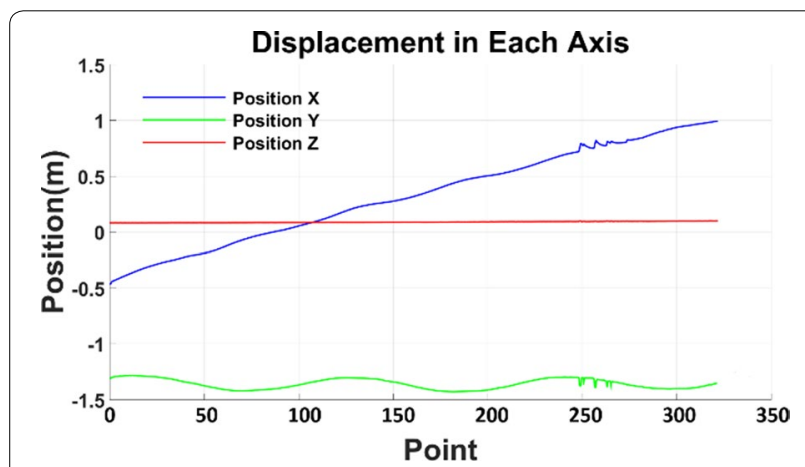

Fig. 12 Snake robot serpentine locomotion displacement in each axis robot doesn't have slide-back either at $\mathrm{X}$ - or $Y$-axis. Figure 13 gives the snake robot trajectory, showing the forward locomotion in the $X$ direction and sinusoidal swing in the $Y$ direction. The calculated trajectory follows the real locomotion which proves the accuracy of IMU tracking system.

To improve snake robot adaptivity in different environments, the sidewinding locomotion mode is developed in this paper. For better locomotion analysis, it also employs a video tracking software. The 6 points video tracking results are shown in the bottom picture of Fig. 14. In

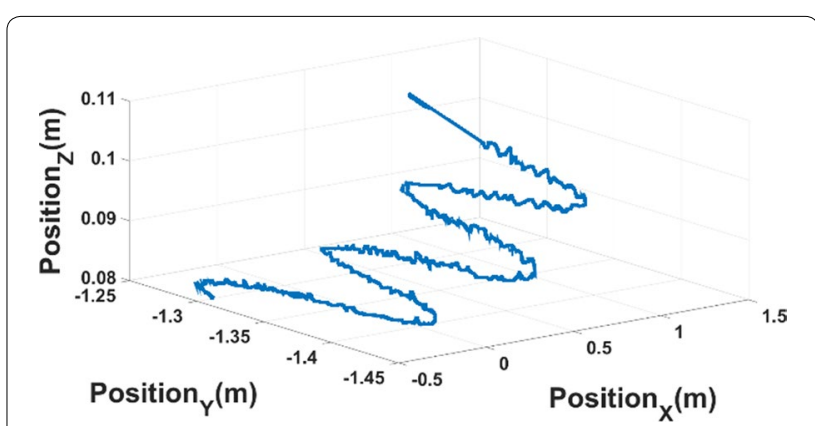

Fig. 13 Snake robot serpentine trajectory

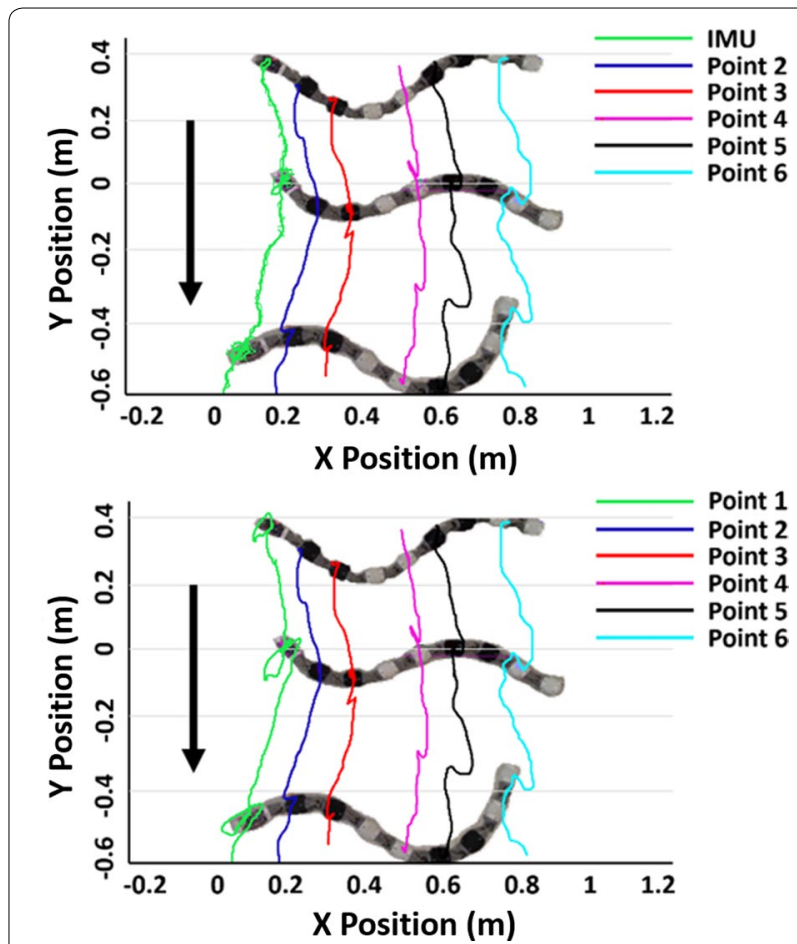

Fig. 14 Tracking result of sidewinding locomotion 

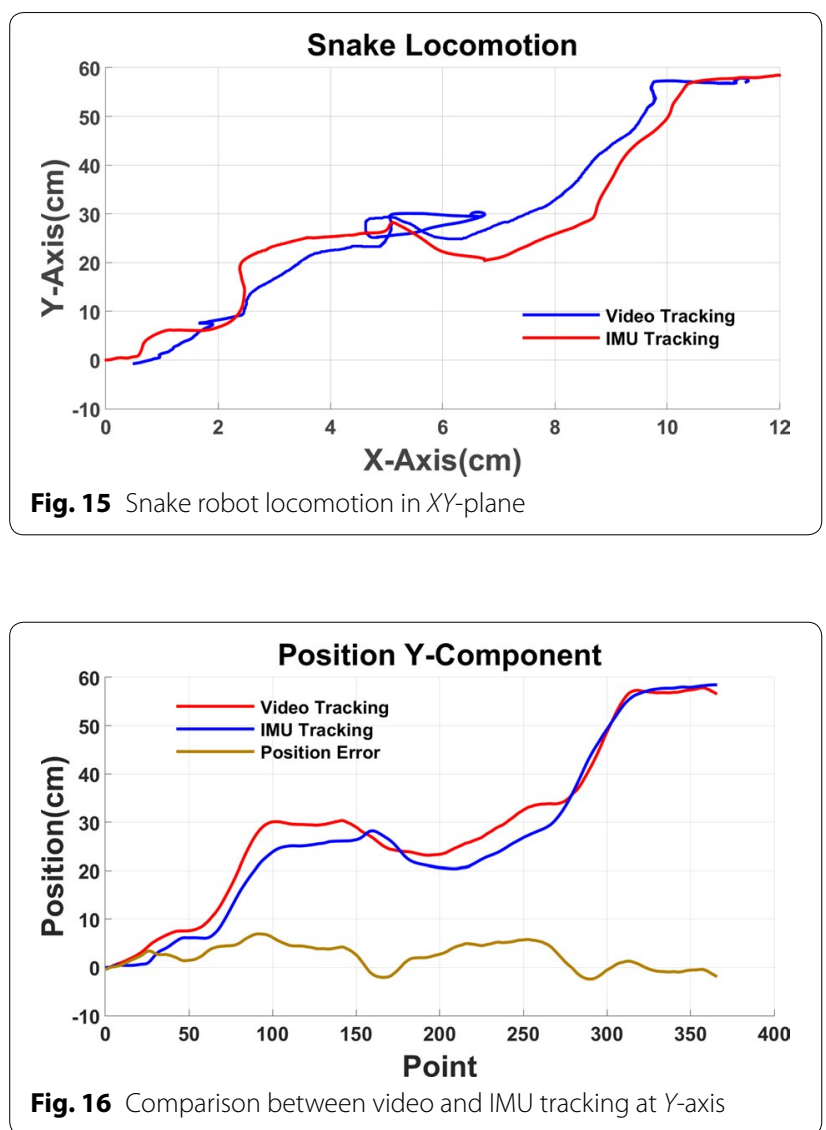

Table 4 Comparison of tracking results

\begin{tabular}{lll}
\hline & Displacement $(\mathbf{c m})$ & Velocity $(\mathbf{c m} / \mathbf{s})$ \\
\hline IMU tracking & 106.85 & 28.59 \\
Video tracking & 95.25 & 25.34 \\
Simulation & 100 & 24.3 \\
\hline
\end{tabular}

the above picture, IMU tracking is used to replace video tracking of point 1 which is shown by the green line. Compared with video tracking result, IMU tracking still has some glitches, thus leading to errors. The error analysis follows.

Let the snake robot move along a straight line. The video recorder is set to be perpendicular to the snake robot, in order to make the video tracking work well. The snake robot's locomotion in $X Y$-plane is shown in Fig. 15. Snake robot's locomotion in $Y$-axis is used to calculate IMU tracking which is shown in Fig. 16. Comparing with the video tracking results, the IMU tracking still has small errors. The results show that the maximum error is $12.23 \%$.

The absolute displacement and average velocity of the two tracking systems are listed in Table 4.

Since the video tracking software has higher accuracy, results of video tracking are used as the reference to calculate the error of IMU. Repeating the experiment to find out the average error of IMU tracking system, the results are shown in Table 5.

Based on the experiments, the average displacement error is $12.01 \%$, and the average velocity error is $9.25 \%$. The error may come from

- Accelerometer accuracy.

- The measurement error due to mechanical characteristics of MEMS under the influence of the environment.

- Gravity.

- Random noise.

\section{Conclusion}

This paper presents a solution to build a snake robot with the tracking system. The tracking system/algorithm can be used for locomotion analysis based on IMU data.

Table 5 Average tracking error from experiments

\begin{tabular}{|c|c|c|c|c|c|c|c|c|}
\hline Num. & $\begin{array}{l}\text { Video Dis. } \\
X \text {-axis }\end{array}$ & $\begin{array}{l}\text { Video Dis. } \\
Y \text {-axis }\end{array}$ & IMU Dis. $X$-axis & IMU Dis. $Y$-axis & $\begin{array}{l}\text { Video Ave. } \\
\text { speed }\end{array}$ & $\begin{array}{l}\text { IMU Ave. } \\
\text { speed }\end{array}$ & $\begin{array}{l}\text { Average veloc- } \\
\text { ity error (\%) }\end{array}$ & Dis. error (\%) \\
\hline 1 & 34.37 & 95.85 & 38.65 & 106.85 & 24.90 & 27.12 & 8.93 & 11.59 \\
\hline 2 & 31.56 & 93.48 & 36.14 & 105.61 & 24.28 & 26.80 & 10.40 & 12.14 \\
\hline 3 & 32.14 & 96.54 & 36.54 & 107.36 & 25.08 & 27.25 & 8.67 & 11.48 \\
\hline 4 & 33.19 & 101.3 & 39.45 & 109.74 & 26.31 & 27.85 & 5.88 & 9.87 \\
\hline 5 & 36.21 & 98.65 & 40.19 & 109.22 & 25.62 & 27.72 & 8.19 & 11.56 \\
\hline 6 & 34.95 & 97.51 & 38.47 & 110.35 & 25.33 & 28.01 & 10.58 & 12.23 \\
\hline 7 & 35.41 & 101.3 & 39.10 & 113.84 & 26.31 & 28.89 & 9.81 & 12.18 \\
\hline 8 & 34.87 & 95.11 & 37.30 & 107.97 & 24.70 & 27.40 & 10.93 & 12.12 \\
\hline 9 & 39.12 & 105.9 & 45.85 & 118.54 & 27.50 & 30.09 & 9.41 & 12.21 \\
\hline 10 & 34.84 & 96.34 & 36.54 & 108.21 & 25.02 & 27.46 & 9.76 & 11.70 \\
\hline
\end{tabular}


Experiments on the algorithm validate its effectiveness. The algorithm can be further improved as the advanced data processing methods.

\section{Author's contributions}

YTS supervised the project. WXYdeveloped the motion tracking algorithm and data processing algorithm, and also designed the experiments and comparative experiments. AB modified the custom-built snake robot. All authors read and approved the final manuscript.

\section{Acknowlegements}

The authors thank Yudong Luo and Na Zhao for helping simulation and implementation of the project.

\section{Competing interests}

Weixin Yang, Bajenov Alexandr and Yantao Shen have no competing interests.

\section{Availability of data and materials}

The data sets supporting the conclusions of this article are available in the Google Drive repository https://drive.google.com/ open?id=0B5zKGavqzH_GVIZxUVo3cnl4bUU

\section{Funding}

The project is partially supported by NASA CAN grant \#NNX13AN15A and NASA RID SEED grant NNX15AI02H.

\section{Publisher's Note}

Springer Nature remains neutral with regard to jurisdictional claims in published maps and institutional affiliations.

Received: 6 October 2017 Accepted: 31 October 2017

Published online: 10 November 2017
References

1. Gray J. The mechanism of locomotion in snakes. J Exp Biol. 1946;23(2):101-120. http://jeb.biologists.org/content/23/2/101.full.pdf

2. Hirose S. Biologically inspired robots: serpentile locomotors and manipulators. Oxford: Oxford University Press; 1993.

3. Ma S, Tadokoro N. Analysis of creeping locomotion of a snake-like robot on a slope. Auton. Robots. 2006;20(1):15-23. https://doi.org/10.1007/ s10514-006-5204-6.

4. Sabatelli S, Galgani M, Fanucci L, Rocchi A. A double-stage kalman filter for orientation tracking with an integrated processor in 9-d imu. IEEE Trans Instrum Measur. 2013;62(3):590-8. https://doi.org/10.1109/ TIM.2012.2218692.

5. Lee JK, Park EJ. Quasi real-time gait event detection using shank-attached gyroscopes. Med Biol Eng Comput. 2011;49(6):707-12. https://doi. org/10.1007/s11517-011-0736-0.

6. Faulkner WT, Alwood R, Taylor DWA, Bohlin J, David D, Taylor WA. Gpsdenied pedestrian tracking in indoor environments using an imu and magnetic compass. Proc Int Tech Meet Inst Navig Itm. 2010;1(2):198-204.

7. Foxlin E. Inertial head-tracker sensor fusion by a complementary separate-bias Kalman filter. In: Proceedings of the IEEE 1996 virtual reality annual international symposium; 1996. pp. 185-194. https://doi. org/10.1109/NRAIS.1996.490527

8. Corrales JA, Candelas FA, Torres F. Hybrid tracking of human operators using IMU/UWB data fusion by a kalman filter. In: 2008 3rd ACM/IEEE international conference on human-robot interaction (HRI); 2008. pp. 193-200. https://doi.org/10.1145/1349822.1349848

9. Zhang P, Gu J, Milios EE, Huynh P. Navigation with IMU/GPS/digital compass with unscented Kalman filter. In: IEEE international conference mechatronics and automation, vol. 3. 2005; pp. 1497-15023 (2005). https://doi.org/10.1109/ICMA.2005.1626777

10. Sabatini AM. Quaternion-based extended kalman filter for determining orientation by inertial and magnetic sensing. IEEE Trans Biomed Eng. 2006:53(7):1346-56. https://doi.org/10.1109/TBME.2006.875664.

11. Speed Integration Principle. http://www.diangon.com/m22602.html

12. Discrete Kalman Filter. http://wolfweb.unr.edu/fadali/EE782/DiscreteKF. pdf

\section{Submit your manuscript to a SpringerOpen ${ }^{\circ}$ journal and benefit from:}

- Convenient online submission

- Rigorous peer review

- Open access: articles freely available online

- High visibility within the field

- Retaining the copyright to your article

Submit your next manuscript at $\boldsymbol{\nabla}$ springeropen.com 